\title{
Caracterização do defeito spalling e correlação com a região da solda aluminotérmica em trilho ferroviário
}

\author{
Gregory de Oliveira Miranda $1 *$ (1) \\ Vinicius Silva dos Reis ${ }^{1}$ \\ Carlos Vinicius de Paes Santos ${ }^{2}$ \\ Fábio Alves Rabelo Júnior ${ }^{3}$ \\ Edgar Costa Cardoso \\ José Maria do Vale Quaresma ${ }^{2}$
}

\section{Resumo}

Dentre os defeitos provenientes de fadiga de contato de rolamento (RCF) nos trilhos ferroviários, o spalling se apresenta como um dos mais severos devido às suas características morfológicas. Este trabalho envolve a análise de um amostra de solda aluminotérmica de trilho ferroviário contendo um defeito do tipo spalling, nas caracterizações buscou-se fazer a correlação entre o defeito, a microestrutura e as propriedades mecânicas na região do trilho. Foram realizados ensaios de dureza e microdureza, além da caracterização microestrutural e fractográfica via microscopia óptica. Os resultados mostraram que o spalling possui uma forte tendência em ocorrer na região da Zona Termicamente Afetada (ZTA), expressa pela variação microestrutural e dureza ao longo da amostra e as trincas são influenciadas pela microestrutura deformada da superfície do trilho.

Palavras-chave: Defeitos em trilhos; Caracterização; Fadiga de contato de rolamento; Spalling.

\section{Characterization of rail spalling defect and correlation with the region of the aluminothermic weld on rail}

\begin{abstract}
Among the defects caused by rolling contact fatigue (RCF) on railways, the spalling is one of the most severe due to its morphological characteristics. This work involves the characterization of a sample containing spalling in a Thermite weld sample, making the correlation between the defect, the microstructure and mechanical properties of the rail region. Hardness and microhardness tests were performed and microstructural characterization was evaliated using Optical Microscopy.The results showed that spalling has a strong tendency to occur in the region of the heat affected zone (HAZ), expressed by the microstructure and by the hardness variation throughout the sample and the cracks are influenced by the deformed microstructure of the rail surface.
\end{abstract}

Keywords: Rail defects; Characterization; Rolling contact fatigue; Spalling.

\section{Introdução}

A fadiga de contato de rolamento e o desgaste são problemas inerentes as operações ferroviárias [1], que estão ligados principalmente às tensões de contato entre a roda e o trilho [2-4]. Nesse sentido, há forte influência desses fatores sobre a qualidade e o custo do modal ferroviário, fato esse que tente a ser acentuado devido ao aumento das velocidades e dos carregamentos, que implicam nas tensões de contato. Diante disso, destaca-se a Fadiga de contato de rolamento (Rolling contact fatigue - RCF) que é um mecanismo crucial de danos e rege a vida em serviço e o custo de manutenção

${ }^{1}$ Faculdade de Engenharia Mecânica, Universidade Federal do Pará - UFPA, Belém, PA, Brasil.

${ }^{2}$ Grupo de Pesquisa em Engenharia de Materiais - GPEMAT, Universidade Federal do Pará - UFPA, Belém, PA, Brasil.

${ }^{3}$ Gerência de Manuteção de Caminhões Mecânicos, Vale S.A., PA, Brasil

${ }^{4}$ Diretoria de Sustentabilidade, BIOPALMA, Vale S.A., PA, Brasil.

*Autor correspondente: gregmirandamail@gmail.com 
de trilhos e rodas ferroviárias [5-7]. Devido ao excesso de tensões decorrentes do contato entre a roda e o trilho, pode ocorrer a iniciação e a propagação de trincas na superfície dos trilhos, as quais podem originar defeitos e fraturas, dentre esses, destaca-se o spalling no boleto [8-12].

De acordo com [13], o spalling na superfície de contato dos trilhos é um defeito típico de RCF que interfere na manutenção da via além de acentuar as interações dinâmicas no sistema roda-trilho. $\mathrm{O}$ defeito surge com alguns milímetros de profundidade na pista de rolamento do trilho evoluindo gradativamente à medida que a roda ferroviária passa sobre sua região de localização $[7,12]$. Esta irregularidade na via causa vibração anormal no veículo ferroviário [13].

Ressalta-se que tanto no surgimento quanto no crescimento do defeito spalling há influência do ratchetting, o qual está ligado com o carregamento imposto na interação roda-trilho, o qual excederá o limite de deformação plástica acumulado no material, que formará uma camada na subsuperfície que fica compactada, apresenta mudanças estruturais e mecânicas, assim percebe-se que o ratchetting possui grande influência sobre a fadiga de contato de rolamento, e consequentemente, sobre os defeitos ocasionados pela mesma [14-16].

A incidência de defeitos e falhas em soldas do tipo aluminotérmica é bem descrito por [17], visto que, durante o processo de soldagem as mudanças microestruturais devido ao gradiente térmico influenciam diretamente nas propriedades mecânicas da região solda, conforme apresentado em $[18,19]$.

Neste trabalho é realizada a caracterização físicometalúrgica de amostra de spalling na superfície de um trilho soldado pelo processo de aluminotermia, possivelmente relacionado com as alterações microestruturais nas proximidades da solda.

\section{Materiais e métodos}

\subsection{Seleção das amostras}

A amostra de spalling selecionada para caracterização é apresentada na Figura 1. É possível observar a extensa remoção de material da pista de rolamento do trilho. Além disso, são observados outros tipos de defeitos como o flaking, que é caracterizado como a descamação de pequenas lascas da superfície do trilho e o defeito head check que se trata de múltiplas trincas no canto da bitola.

O seccionamento das amostras do spalling foi realizado da seguinte forma: primeiramente realizou-se o corte por eletroerosão, a fim de extrair a amostra contendo o defeito do topo do boleto (Figura 2A), a fim de facilitar a manipulação e permitindo os cortes mais preciso que foram realizados. Em seguida obteve-se amostras menores (Figura 2B), os cortes foram realizados por meio da cortadora metalográfica cut-off, na qual houve a extração de seções longitudinais L1 e L2 do defeito, identificadas na Figura 2.

\subsection{Ensaios de dureza}

Foram realizados ensaios de dureza na escala Rockwell C na região abaixo do spalling e posteriormente os resultados foram convertidos para dureza na Escala Brinell, conforme recomendado por [20]. Foi obtido um perfil longitudinal de dureza na amostra que inicia a medição na região central do cordão de solda e se estende até o final da ZTA. O espaçamento entre as indentações foi de $5 \mathrm{~mm}$.

\subsection{Caracterização da estrutural}

As seções longitudinais denominadas L1 e L2 foram embutidas a frio com resina epóxi, lixadas com lixas de carbeto de silício nas granulometrias $220,320,400$ e $600 \#$ e polidas utilizando pasta de diamante na sequência 6,3 e $1 \mu \mathrm{m}$. Importante que antes do polimento as amostras sejam limpas utilizando álcool etílico em uma cuba ultrassônica por um tempo de, aproximadamente, 7 minutos para remoção de resquícios de lixa presos e demais contaminantes contidos nas trincas.

A caracterização das trincas do spalling foi realizada com as amostras polidas, sem ataque químico, de modo a analisar como as trincas se propagam no material. Foram obtidas imagens utilizando microscopia óptica com magnificação de 50x.

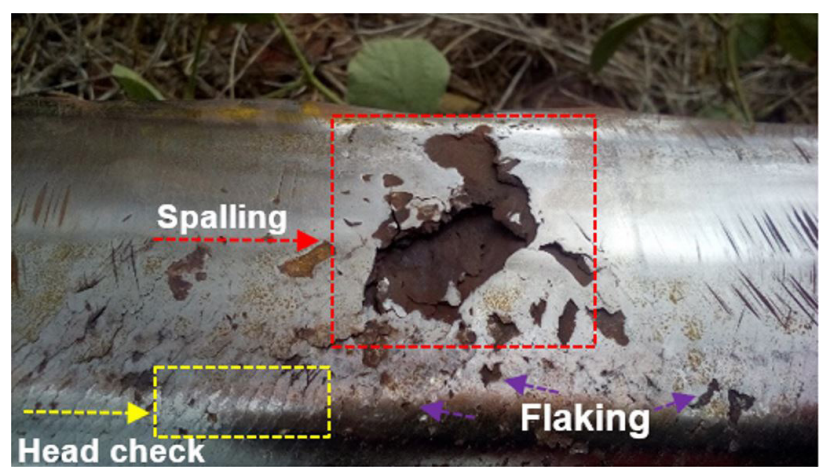

Figura 1. Amostra de trilho removido da via devido a severidade de defeitos superficiais.

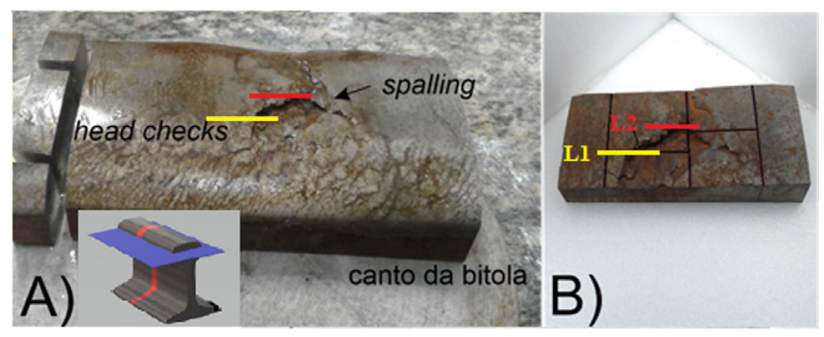

Figura 2. Obtenção de amostras de defeitos em trilhos. (A) Topo do boleto obtido por eletroerosão; (B) amostra de spalling cortada com cortadora metalográfica cut-off. 
A etapa posterior correspondeu à realização do ataque químico por imersão, com reagente Nital 3\% pelo tempo de 5 segundos e a verificação da microestrutura das seções longitudinais via microscopia ótica para obtenção de imagens com aumentos de 50, 100 e 200x.

Para a mensuração da microdureza utilizou-se uma carga de $300 \mathrm{~g}$, a fim de obter os perfis dureza variando desde a superfície até $3,0 \mathrm{~mm}$ de profundidade (com incrementos de $0,25 \mathrm{~mm}$ ). Outrossim, foram realizadas medições nas vizinhanças das trincas subsuperficiais.

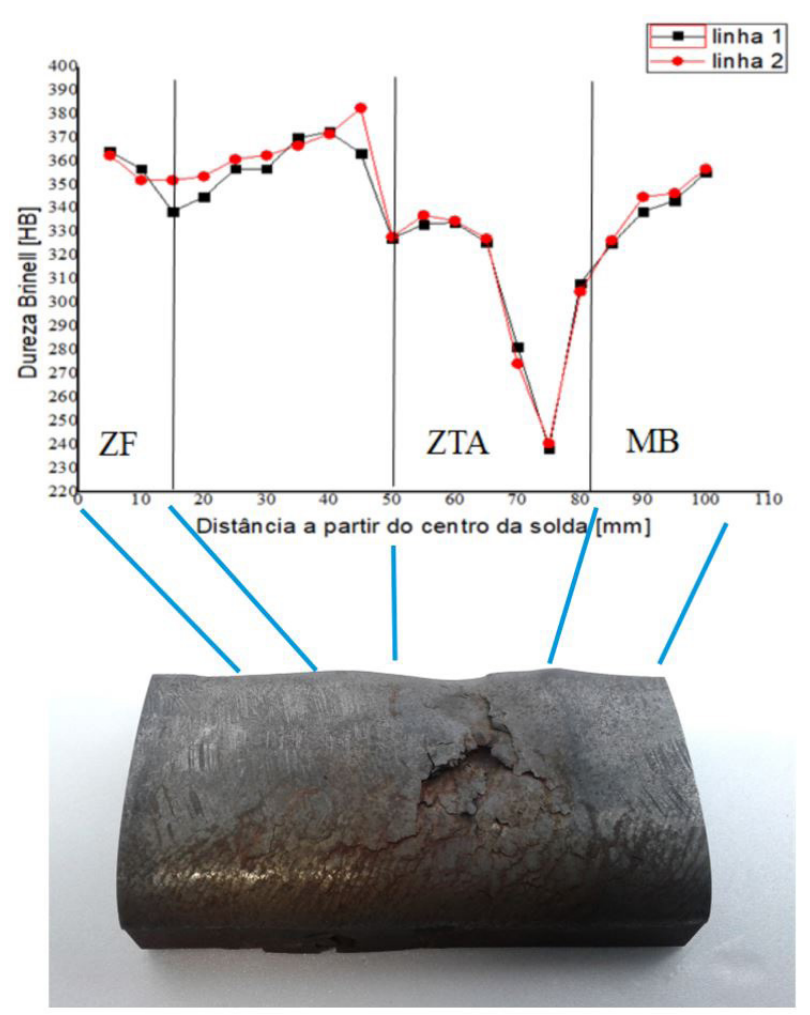

Figura 3. Morfologia dos defeitos no trilho e perfil longitudinal de dureza: zona de fusão (ZF), zona termicamente afetada (ZTA) e metal base (MB).

\section{Resultados}

\subsection{Ensaios de dureza}

O perfil longitudinal de dureza (Figura 3 ) exibe um comportamento variado, uma vez que se trata da região próxima à solda aluminotérmica. A primeira região $(\mathrm{ZF})$ localiza-se onde é depositada a mistura aluminotérmica. Entre 50 e $80 \mathrm{~mm}$ é observada uma redução nos valores, atingindo seu mínimo no final da região. A justificativa relaciona-se com a zona termicamente afetada - ZTA, a qual exibe menores valores de resistência mecânica perfil de dureza notado nos trabalhos de Merıç et al. [21] e Alves et al. [22]. Posterior à esta região, os valores crescem novamente, até atingir a dureza média do metal de base (trilho não afetado pela soldagem).

\subsection{Caracterização das trincas do spalling}

Na Figura 4 é apresentada a fractografia superficial do spalling, identificando as seções longitudinais que foram extraídas. Observa-se que essas trincas se iniciam na superfície e propagam-se em direção ao interior do trilho, não havendo grandes mudanças no ângulo de propagação. As trincas do defeito se propagam com profundidades médias entre 2,3 e $4,2 \mathrm{~mm}$ com ângulos entre $12^{\circ} \mathrm{e} 14^{\circ}$.

Ademais, observa-se que durante a propagação das trincas, alguns trechos acima da trinca sofrem fratura vertical (Figura 5). Dessa forma, uma porção da superfície do trilho é removida na forma de lascas. Essa remoção de material, progressivamente, gera a cratera do spalling na superfície do trilho.

As trincas verticais encontradas podem ser originadas a partir do carregamento vertical exercido durante passagem da roda ferroviária que afeta o defeito. A Figura 6 apresenta diferentes configurações de carregamentos sobre corpos em rolamento, além do resultado que influencia na formação das trincas superficiais e subsuperficiais.
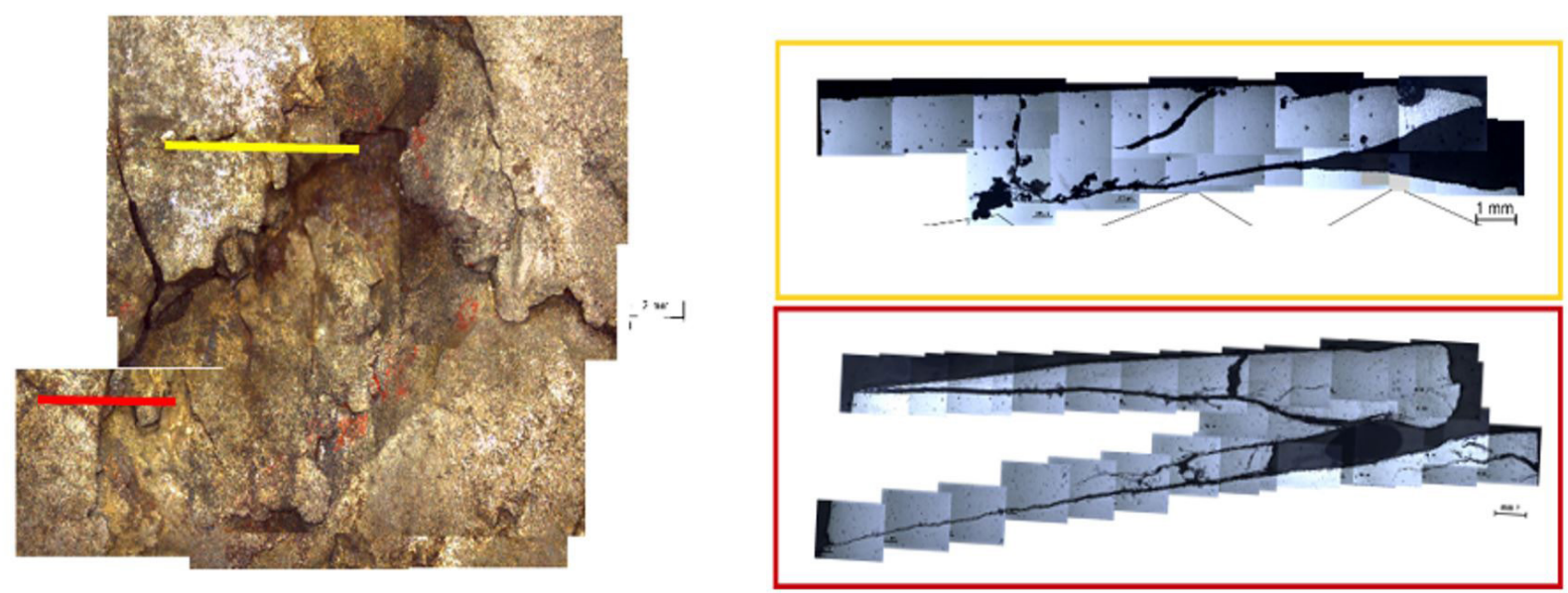

Figura 4. Seções longitudinais L1 (amarelo) e L2 (vermelho) do spalling. 

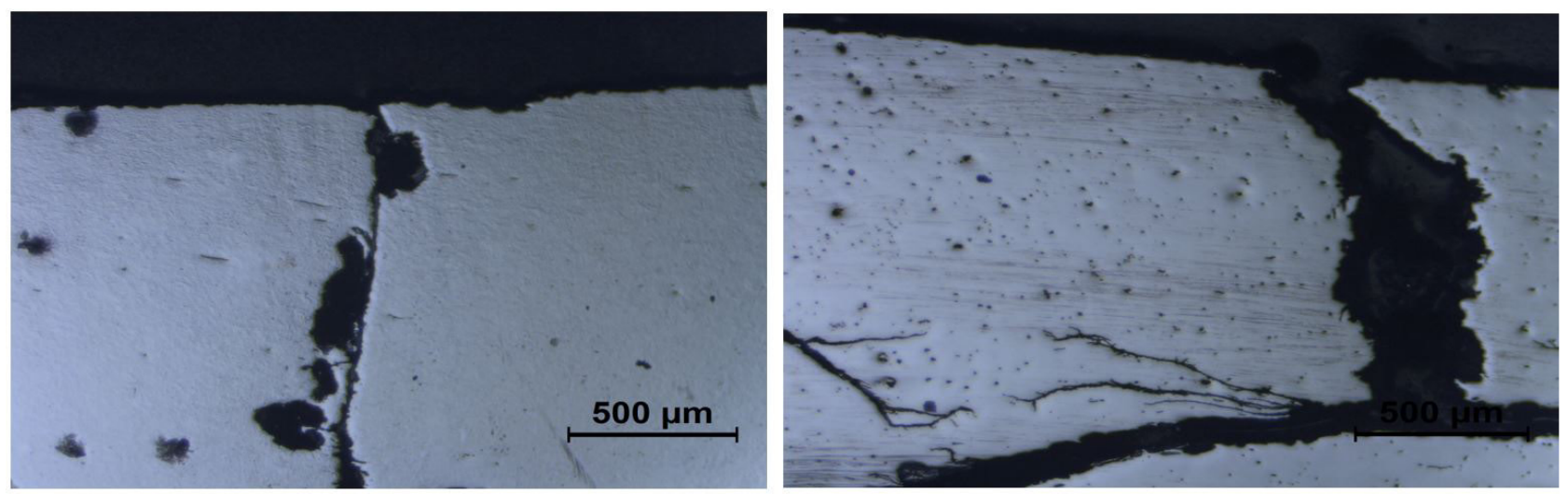

Figura 5. Trincas verticais nas seções L1 e L2, respectivamente, as quais são causadoras do spalling.

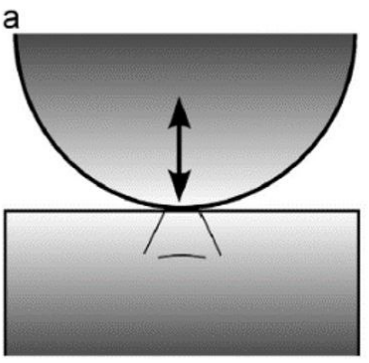

d

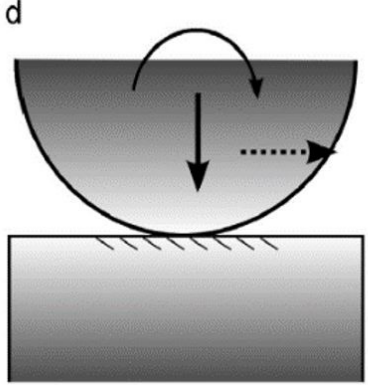

b

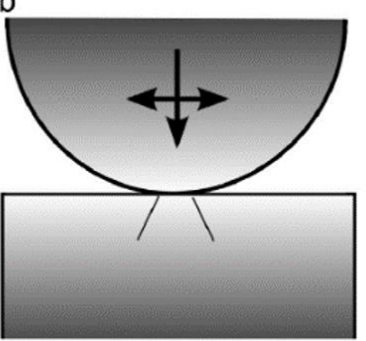

e

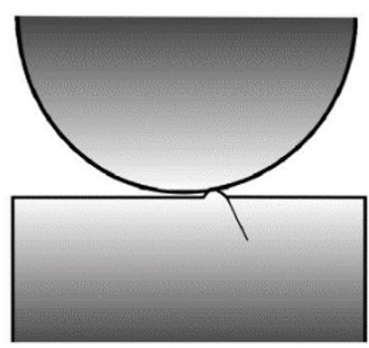

Figura 6. Configurações do contato de corpos em rolamento e a influência na formação de trincas de RCF: a) Carregamento vertical pusante; b) Carregamento vertical com oscilação lateral; c) Rolamento com carregamento vertical; d) Rolamento com carregamento vertical e cisalhamento; e) Presença de irregularidade na superfície de contato. Fonte: Ekberg et al. [24].

Na Figura 6a o carregamento vertical, pulsante pode originar trincas verticais e/ou subsuperficiais. $\mathrm{Na} 6 \mathrm{~b}$ ocorre oscilação lateral e vertical originando apenas trincas superficiais verticais. Já na Figura 6c ocorre a situação de um corpo em rolamento ser pressionado por um carregamento vertical, o que pode originar trincas as subsuperficiais. Se essa configuração é acompanhada de cisalhamento e de deslizamento (devido à frenagem ou à tração), pode ocorrer o surgimento de deformação plástica superficial como mostrado na Figura 6d. A presença de concentradores de tensão na superfície do trilho pode originar trincas superficiais conforme 6e [23].

\subsection{Avaliação microestrutural}

A partir da avaliação microestrutural, observou-se na superfície do trilho uma camada, na qual a microestrutura apresenta-se deformada, isto é, com menores tamanhos de grãos. Nesse contexto, a região em questão é denominada ratchetting, a qual é resultante das tensões impostas pelo carregamento proveniente das rodas ferroviárias, assim induzindo esforços trativos e taxas de deslizamento que causam o trabalho a frio na superfície do trilho [24]. Na Figura 7 é feita a comparação da microestrutura na superfície, a cerca de $0,2 \mathrm{~mm}$ de profundidade, além de mais abaixo, ou seja, cerca de $2 \mathrm{~mm}$.

Na Figura 8 é mostrado como o ratcheting influencia na propagação das trincas do spalling, uma vez que a trinca se propaga na interface da camada deformada e da não deformada $[15,16]$. Acima da trinca é possível visualizar a direção do alinhamento da microestrutura e abaixo a microestrutura apresenta-se mais dispersa.

A partir das imagens de microscopia óptica da seção L1, como visto na Figura 8A, foi possível observar a variação microestrutural longitudinalmente. A consideração a ser feita diz respeito ao fato de que o spalling situa-se na região próximo à solda aluminotérmica região de decrescimento da região e com tendência de defeitos [22]. Dessa forma, o calor gerado durante o processo induz alterações microestruturais, inclusive o processo de esferoidização parcial no final da 
ZTA Inter crítica que pode gerar redução na dureza, exposto nos trabalhos de Akselsen et al. [25] e Liu et al. [26].

$\mathrm{O}$ defeito inicia e propaga-se longitudinalmente com origem na superfície do trilho não afetado pelo calor da soldagem, apresentando microestrutura perlítica até atingir uma microestrutura mais refinada, situada na ZTA, conforme evidenciado pelo direcionamento apresentado na Figura 9.
Conforme observado na Figura 10 percebe-se que na superfície são encontrados maiores valores de microdureza Vickers, e também, é observado que na vizinhança da trinca ocorre uma ligeira redução dos valores de dureza.

Os resultados de microdureza varrendo desde a superfície mostram valores mais acentuados (com pico de $410 \mathrm{HV})$ no início e redução dos valores atingindo a
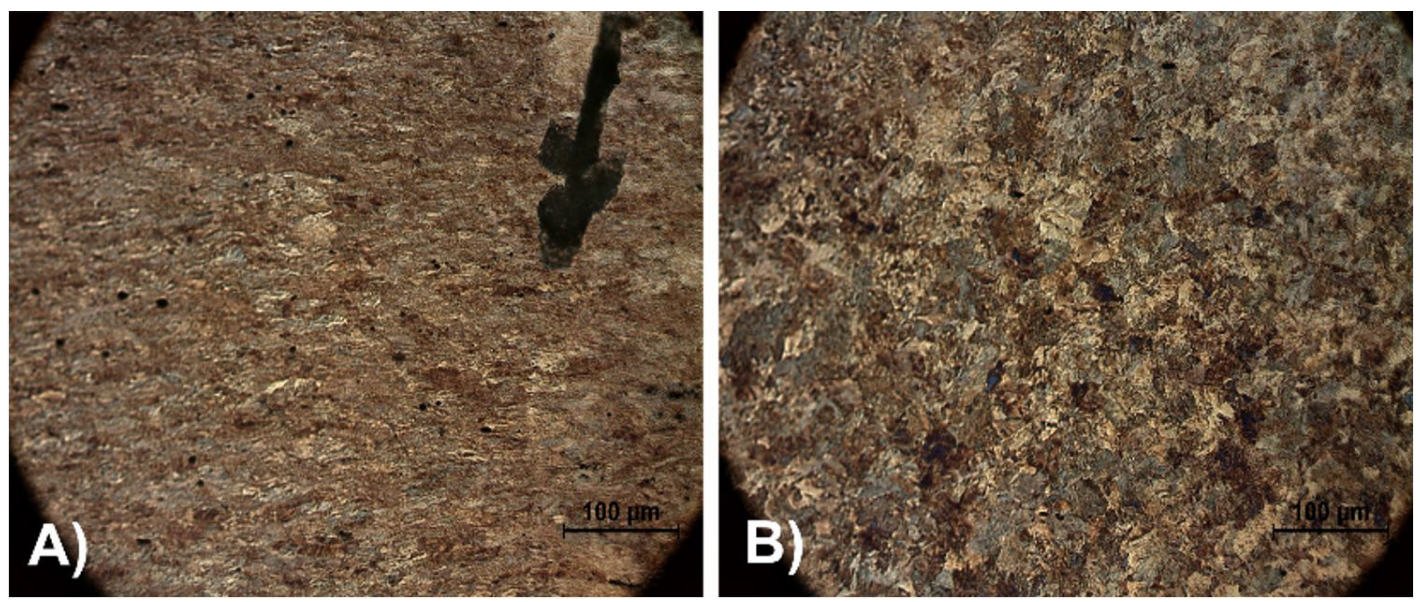

Figura 7. Comparação da microestrutura na profundidade: (A) de 0,2 mm; e (B) de $2 \mathrm{~mm}$.
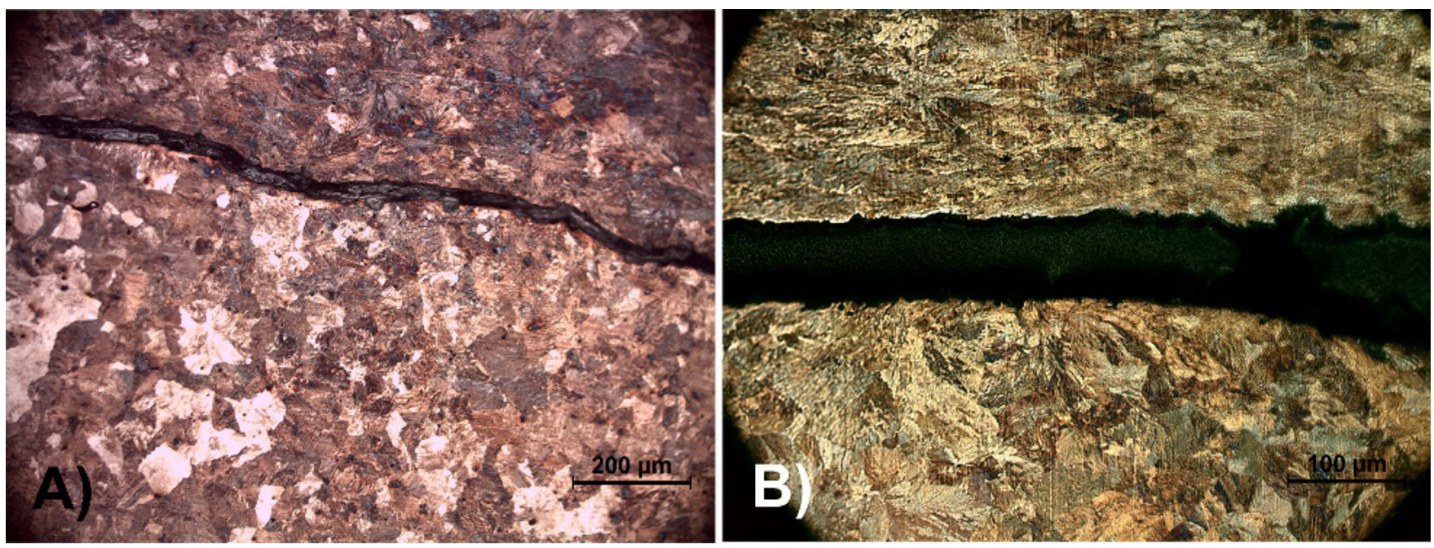

Figura 8. Influência do ratcheting na propagação da trinca. (A) Trinca na seção L1, magnificação de 100x; (B) Seção L2, magnificação de 200x.

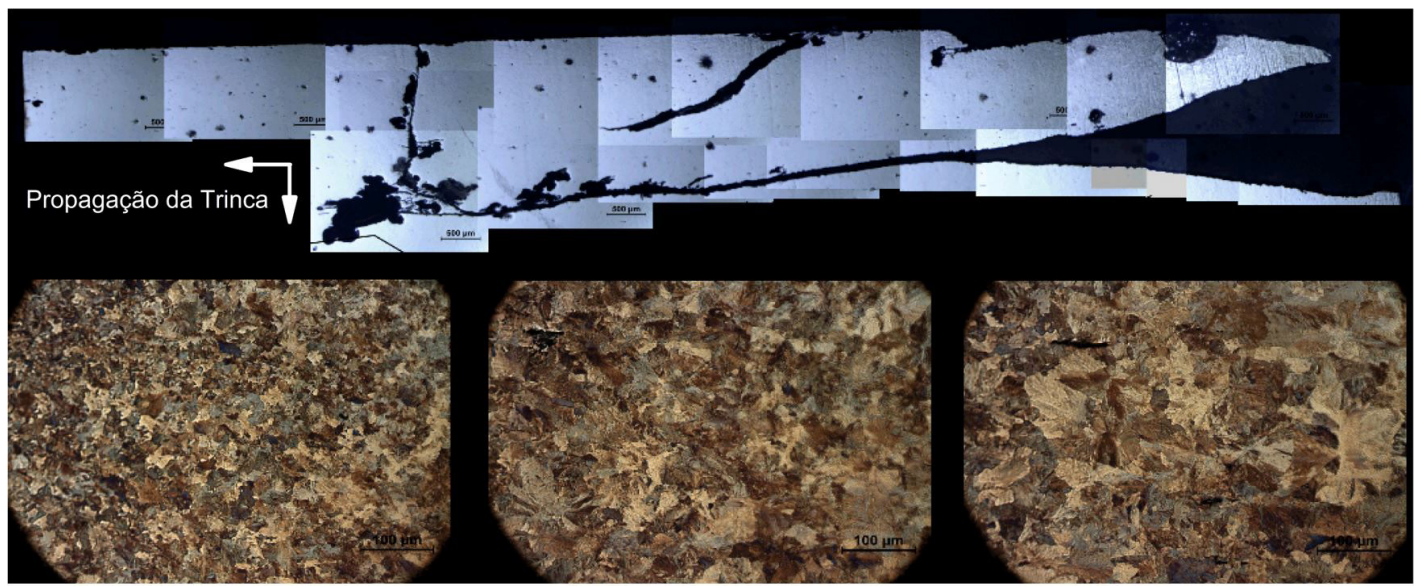

Figura 9. Variação microestrutural ao longo do spalling na seção longitudinal L1. À microestrutura mais à esquerda situa-se no final da ZTA, sendo mais refinada que as demais. 


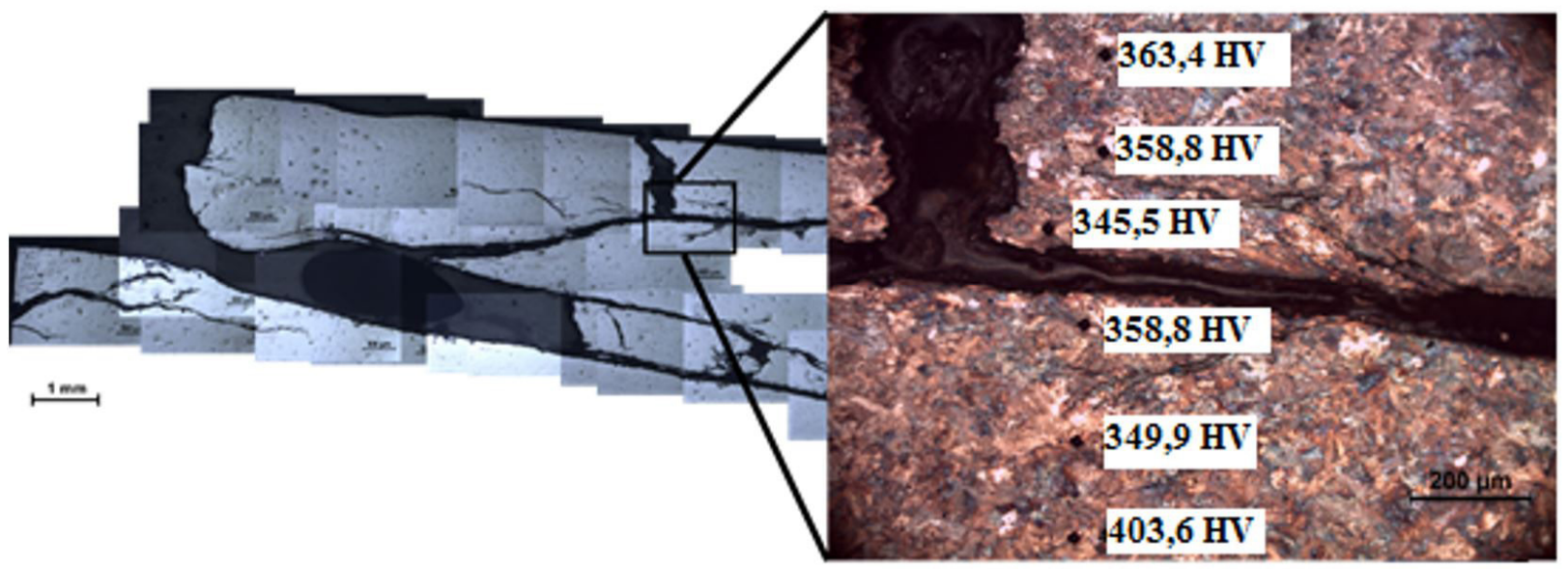

Figura 10. Perfil de microdureza Vickers nas vizinhanças de uma trinca de spalling.

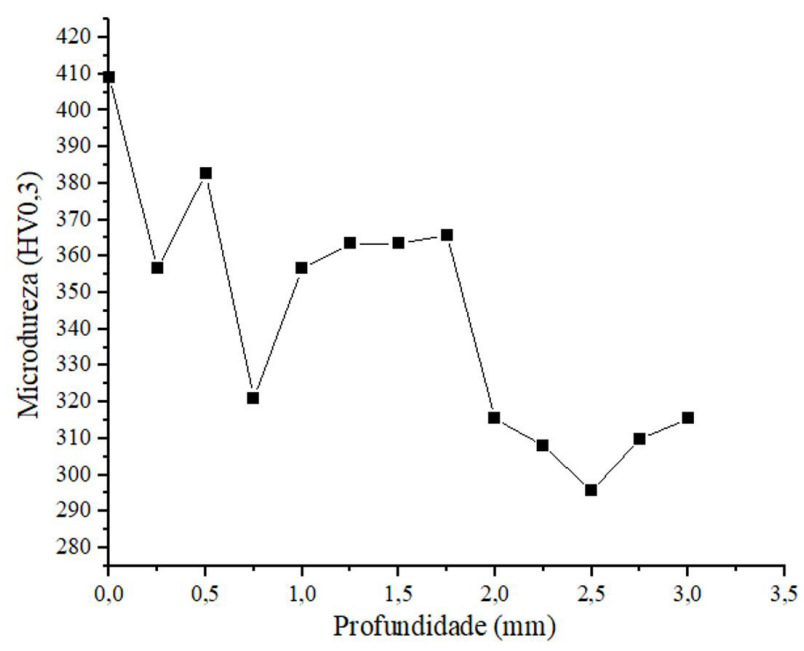

Figura 11. Perfil de microdureza Vickers da superfície até $3,0 \mathrm{~mm}$ de profundidade.

microdureza média do trilho $(350 \mathrm{HV})$ a partir de $2,0 \mathrm{~mm}$ (Figura 11). A superfície sofre maior quantidade de trabalho a frio devido ao intenso contato com a roda, isto é, apresentará maior acumulo de deformação plástica aliado a mudanças microestruturais que influenciaram na propriedade mecânica em questão [27].

\section{Conclusões}

De acordo com a metodologia adotada e as discussões dos resultados obtidos, as seguintes conclusões foram obtidas: i. As características do defeito spalling evidenciam que o mesmo apresenta grande severidade na ferrovia e a sua remediação deve ser imediata, tendo em vista que o seu crescimento desenfreado pode acarretar em danos maiores no trilho e na roda ferroviária;

ii. O spalling apresenta trincas iniciadas na superfície com ângulos entre 12 e $14^{\circ}$, que se propagam até certas profundidades, nas quais por diversos fatores há o surgimento de uma trinca vertical que causa a remoção de material;

iii. Foi observada a influência do ratchetting que ocorre nas camadas superiores do trilho na propagação das trincas do spalling. $\mathrm{O}$ defeito se propaga preferencialmente na interface entre a camada deformada e a não-deformada;

iv. A localização do defeito, particularmente na amostra estudada, corresponde à região da zona termicamente afetada (ZTA) originada da soldagem aluminotérmica. Esta região possui cerca de $30 \mathrm{~mm}$ de largura e as trincas do spalling se iniciam na região fora da ZTA e crescem em direção a mesma. $\mathrm{Na}$ avaliação microestrutural foi observada a diferença das microestruturas.

\section{Agradecimentos}

Os autores gostariam de agradecer à VALE S.A. pela iniciativa do projeto Cátedra Roda-Trilho e apoio financeiro ao desenvolvimento deste trabalho.

\section{Referências}

1 Reddy V, Chattopadhyay G, Larsson-Kråik PO, Hargreaves DJ. Modelling and analysis of rail maintenance cost. International Journal of Production Economics. 2007;105(2):475-482.

2 Lewis R, Olofsson U. Wheel-rail interface handbook. 1st ed. Washington: Woodhead Publishing; 2009. 
3 Seo JW, Jun HK, Kwon SJ, Lee DH. Rolling contact fatigue and wear of two different rail steels under rollingsliding contact. International Journal of Fatigue. 2016;83:184-194.

4 Magel E, Mutton P, Ekberg A, Kapoor A. Rolling contact fatigue, wear and broken rail derailments. Wear. 2016;366367:249-257.

5 Ekberg A, Kabo E, Andersson H. An engineering model for prediction of rolling contact fatigue of railway wheels. Fatigue \& Fracture of Engineering Materials \& Structures. 2002;25(10):899-909.

6 Zerbst U, Mädler K, Hintze H. Fracture mechanics in railway applications: an overview. Engineering Fracture Mechanics. 2005;72(2):163-194.

7 Steenbergen M. Rolling contact fatigue: spalling versus transverse fracture of rails. Wear. 2017;380-381:96-105.

8 Ekberg A, Kabo E, Andersson H. An engineering model for prediction of rolling contact fatigue of railway wheels. Fatigue \& Fracture of Engineering Materials \& Structures. 2002;25(10):899-909.

9 Ekberg A, Kabo E, Nielsen JC, Lundén R. Subsurface initiated rolling contact fatigue of railway wheels as generated by rail corrugation. International Journal of Solids and Structures. 2007;44(24):7975-7987.

10 Seo J, Kwon S, Lee D. Effects of surface defects on rolling contact fatigue of rail. Procedia Engineering. 2011;10:1274-1278.

11 Reis L, Li B, De Freitas M. A multiaxial fatigue approach to Rolling Contact Fatigue in railways. International Journal of Fatigue. 2014;67:191-202.

12 Yang R, Cao S, Kang W, Li J, Jiang X. Mechanism analysis of spalling defect on rail surface under rolling contact conditions. Mathematical Problems in Engineering. 2018;2018:7012710.

13 Wang K, Zhai W, Lv K., Chen Z. Numerical investigation on wheel-rail dynamic vibration excited by rail spalling in high-speed railway. Shock and Vibration. 2016;2016:9108780

14 Franklin FJ, Kapoor A. Modelling wear and crack initiation in rails. Proceedings of the Institution of Mechanical Engineers. Part F, Journal of Rail and Rapid Transit. 2007;221(1):23-33.

15 Kapoor A. Wear by plastic ratchetting. Wear. 1997;212(1):119-130.

16 Mazzù A, Donzella G. A model for predicting plastic strain and surface cracks at steady-state wear and ratcheting regime. Wear. 2018;400-401:127-136.

17 Mutton P, Cookson J, Qiu C, Welsby D. Microstructural characterisation of rolling contact fatigue damage in flashbutt welds. Wear. 2016;366-367:368-377.

18 Karguin VA, Tikhomirova LB, Galay MS, Kuznetsova YS. Improving service properties of welded joints produced by aluminothermic welding. Welding International. 2015;29(2):155-157.

19 Sarikavak Y, Turkbas OS, Cogun C. Influence of welding on microstructure and strength of rail steel. Construction \& Building Materials. 2020;243:118220.

20 American Railway Engineering and Maintenance-of-Way Association. Manual for railway engineering. Lanham: AREMA; 2010.

21 Merıç C, Atık E, Şahın S. Mechanical and metallurgical properties of welding zone in rail welded via thermite process. Science and Technology of Welding and Joining. 2002;7(3):172-176.

22 Alves LHD, Tepedino TC, Masoumi M, Tressia G, Goldenstein H. Metallurgical and tribological aspects for squat formation in the aluminothermic weld HAZ edges of rails welded using aluminothermy. Industrial Lubrication and Tribology. 2020;72(9):1123-1131.

23 Seo JW, Jun HK, Kwon SJ, Lee DH. Rolling contact fatigue and wear of two differents rail steels under rollingsliding contact. International Journal of Fatigue. 2016;83:184-194.

24 Ekberg A, Akesson B, Kabo E. Wheel/rail rolling contact fatigue: probe, predict, prevent. Wear. 2014;314(1-2):2-12.

25 Akselsen OM, Grong Ø, Solberg JK. Structureproperty relationships in intercritical heat affected zone of lowcarbon microalloyed steels. Materials Science and Technology. 1987;3(8):649-655.

26 Liu Y, Tsakadze Z, Hoh HJ, Pang JHL, Christian I, Ng TX, et al. Mechanical properties and microstructural analysis of rail thermite welding joints. In: 2018 International Conference on Intelligent Rail Transportation (ICIRT); 2018; Singapore. New York: IEEE; 2018. p. 1-4.

27 Dylewski B, Risbet M, Bouvier S. The tridimensional gradient of microstructure in worn rails-experimental characterization of plastic deformation accumulated by RCF. Wear. 2017;392-393:50-59.

Recebido em: 27 Maio 2020

Aceito em: 19 Out. 2020 